\title{
Effects of different initial condition samplings on photodynamics and spectrum of pyrrole
}

\author{
Mario Barbatti ${ }^{1}$ and Kakali Sen ${ }^{2}$ \\ ${ }^{1}$ Aix Marseille Université, CNRS, ICR UMR7273, 13397 Marseille, France. \\ ${ }^{2}$ Department of Chemistry, École Normale Supérieure, UMR ENS-CNRS-UPMC 8640, 24 Rue \\ Lhomond, 75005 Paris, France. \\ Correspondence to: Mario Barbatti (Email: mario.barbatti@univ-amu.fr)
}

\begin{abstract}
Semi-classical simulations of spectrum and dynamics of complex molecules require statistical sampling of coordinates and momenta. The effects of using thermal and quantum samplings are analyzed taking pyrrole as a test case. It is shown that there are significant differences in the results obtained with each of these two approaches. Overall, quantum sampling based on a Wigner distribution renders superior results, comparing well to the experiments. Dynamics simulations based on surface hopping and $\mathrm{ADC}(2)$ reveal that pyrrole internal conversion to the ground state occurs not only through H-elimination path, but also through ring-distortion paths, which have been systematically neglected by diverse experimental setups. The analysis of the reaction paths also shows that the ionization potential varies by more than $5 \mathrm{eV}$ between ionization of the excited state at the Franck-Condon region and at the intersections with the ground state. This feature may have major implications for time-resolved photoelectron spectroscopy.
\end{abstract}




\section{Graphical abstract}

The effects of using thermal and quantum samplings to create initial conditions for dynamics and spectrum simulations are analyzed for pyrrole. There are significant differences in the results obtained with each of these two approaches. Overall, quantum sampling based on a Wigner distribution renders superior results compared to the experiments.

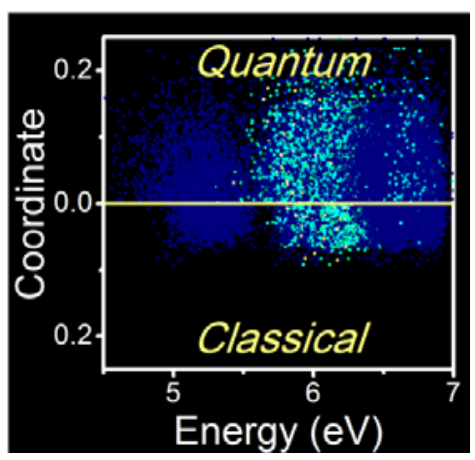




\section{Introduction}

In diverse types of simulations, one needs to sample nuclear conformations of a molecule considering the kinetic energy deposited in the internal vibrational modes. This is the case, for instance, of the sampling of the phase space (geometries and velocities) to initiate excited-state dynamics simulations or the sampling of the configurational space (geometries only) for spectrum simulations. There are two basic approaches to carry out these samplings: one may either sample the space by considering that the molecule is at its zero-point level (we will refer to it as the quantum or Q sampling); or, alternatively, one may sample the space supposing that the molecule is in thermal equilibrium with the environment (thermal or T sampling).

Although we can find examples of both approaches in the literature, they are far from equivalents. While in the Q sampling each vibrational degree of freedom $j$ has energy $\varepsilon_{v Q}=\hbar \omega_{j} / 2$, in the $\mathrm{T}$ sampling each degree has $\varepsilon_{v T}=k T$. Because at $T=300 \mathrm{~K}, \varepsilon_{v Q}\left(\right.$ roughly, $\left.\left\langle\varepsilon_{v Q}\right\rangle \sim 0.1 \mathrm{eV}\right)$ is much larger than $\varepsilon_{v T}(\sim 0.03 \mathrm{eV}$, see Figure 1-top), this means that simulations based on one or other approach may render different results.

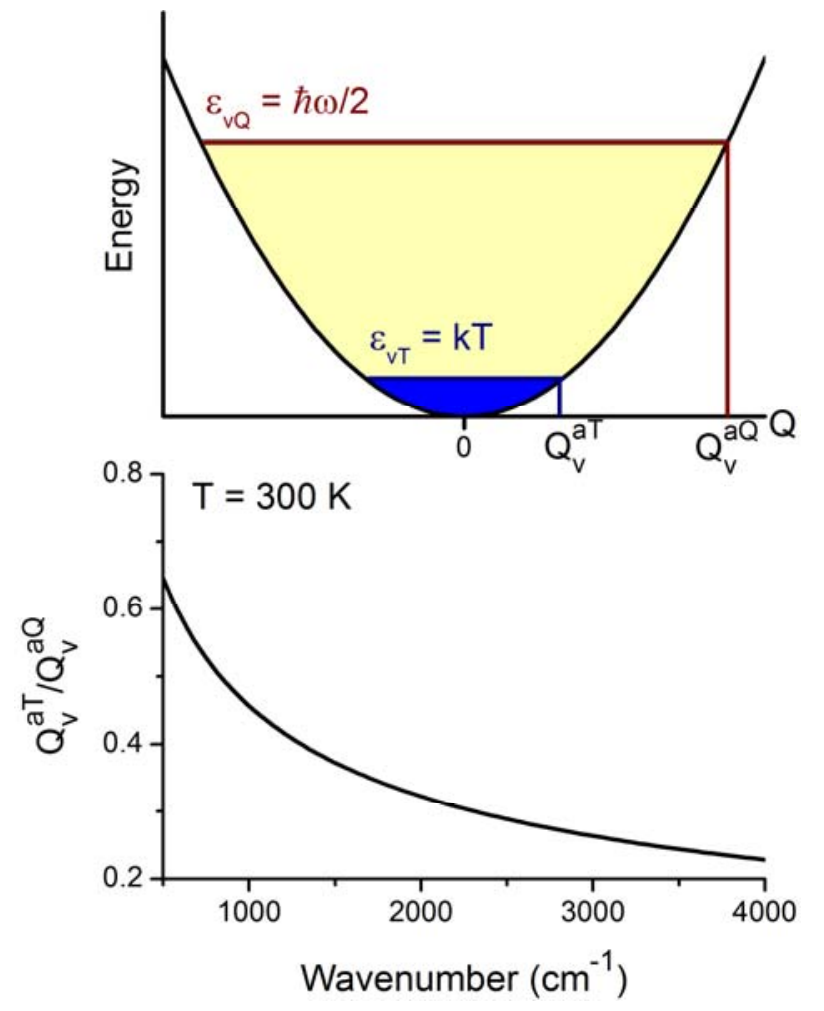

Figure 1. Top: Illustration of the classical $(T=300 \mathrm{~K})$ and quantum energies and amplitudes in a onedimensional harmonic potential. Bottom: Ratio between the classical and quantum amplitudes as a function of the wavenumber (Eq. (1)). The proportions in the upper figure correspond to $1600 \mathrm{~cm}^{-1}$. 
This difference of energy per degree of freedom reflects on the vibrational amplitude. Considering that the amplitude of a harmonic oscillator with energy $\varepsilon$ is $Q^{a}=\left(2 \varepsilon / \mu \omega^{2}\right)^{1 / 2}$, the ratio between the amplitudes in the thermal and quantum samplings is

$$
\frac{Q^{a T}}{Q^{a Q}}=\left(\frac{2 k T}{\hbar \omega_{j}}\right)^{1 / 2} .
$$

In particular, Eq. (1) implies that the deviation between the thermal and quantum amplitudes tends to increase for larger wavenumbers (Figure 1-bottom).

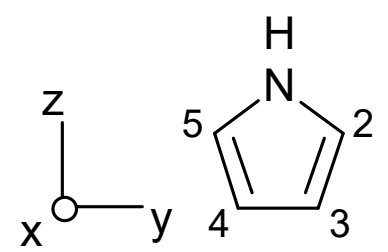

Scheme 1. 1H-Pyrrole.

In this work, we take pyrrole (Scheme 1) as a test case for investigating the differences between the $\mathrm{Q}$ and $\mathrm{T}$ samplings. Pyrrole is a small molecule with ultrafast photodynamics. ${ }^{1}$ This makes it ideal for benchmarking ${ }^{2-10}$ as well as for concept and method developments. ${ }^{11-14}$ Moreover, after high-energy excitation into the bright $\pi \pi^{*}$ state, pyrrole dynamics branches in several distinct reaction paths, ${ }^{15}$ facilitating the comparison and evaluation of the sampling effects. The ultrafast dynamics of photoexcited pyrrole has also been the subject of a large number of experimental (time $^{1,16-22}$ and frequency ${ }^{23-25}$ domains) and theoretical investigations ( statics $^{26-28}$ and dynamics ${ }^{29-35}$ ). We have ourselves taken pyrrole before to investigate dynamics branching, ${ }^{36,37}$ to test methodology, ${ }^{38}$ and to understand the role of the conical intersection branching space on the photoproducts. ${ }^{39}$ Here, we will use it for simulating spectrum and excited-sate dynamics starting from $\mathrm{Q}$ and $\mathrm{T}$ samplings, aiming at understanding their impact on the simulations.

The difference between the $\mathrm{Q}$ and $\mathrm{T}$ samplings has not gone unnoticed, although it has been largely neglected in the field. As far as we know, the only work comparing them in the context of photochemistry is in the paper by Klaffki et al., ${ }^{40}$ where they analyzed the results of excited-state dynamics simulations for a protonated Schiff base (PSB3) based on initial conditions generated with both quantum and thermal samplings. They observed a slight trend towards faster reactions when using Q sampling, although the differences were not really statistically significant. They finally prescribed the use of thermal sampling as the preferred methodology, as the Q sampling would provide "artificially high kinetic energies" and lower numerical stability. 
We tend to disagree with this analysis. The high kinetic energies of the $\mathrm{Q}$ sampling are not artificial, but correspond to the kinetic energies expected for a quantum system. In conceptual terms, the Q sampling through a Wigner distribution should be the proper way of mapping quantum densities on the classical phase space. ${ }^{41}$ From a practical perspective, we have observed before ${ }^{42}$ that while spectra simulated with Q sampling rendered satisfactory results, those based on T sampling were too narrow, needing much larger nominal temperatures to reach proper band width description. We have even proposed ${ }^{43,44}$ a hybrid method to sample initial conditions for QM/MM surface hopping dynamics based on $\mathrm{Q}$ sampling for the QM and T sampling for the MM regions. The present results for spectrum and dynamics will add to these previous evidences of the superiority of $\mathrm{Q}$ over $\mathrm{T}$ samplings.

The main contribution of the present work is at providing a more complete and systematic investigation of the effects of sampling on electronic spectrum and nonadiabatic excited-state dynamics. Although the effects of sampling will most likely depend on each particular molecule, we try to figure out features that may reflect general trends of the different sampling methods. We also aim at establishing protocols for each step of the simulations for evaluating the sampling effects, which may be used in other cases as well. As a side-product of this methodological investigation, we also report a new set of excited-state dynamics for pyrrole in the gas phase.

\section{Computational details}

\section{Electronic Structure and Dynamics}

Excited states were computed with the algebraic diagrammatic construction to the second order $[\mathrm{ADC}(2)],{ }^{45,46}$ using the resolution-of-the-identity (RI) approximation ${ }^{47}$ and frozen $1 \mathrm{~s}$ orbitals. The reference ground state was computed at the second-order Møller-Plesset Perturbation Theory (MP2). ${ }^{48}$ Calculations were done with the aug-cc-pVDZ basis set assigned to for $\mathrm{N}$ and $\mathrm{C}$ atoms, and with the cc-pVDZ assigned to $\mathrm{H}$ atoms. ${ }^{49}$

The absorption spectrum and initial conditions for dynamics were simulated with the nuclear ensemble method ${ }^{50}$ using different types of sampling as discussed below. On-the-fly dynamics simulations were carried out in the excited states computed with $\mathrm{ADC}(2)$. Nonadiabatic effects were taken into account by the surface hopping approach. ${ }^{51}$ Classical equations were integrated with $0.5 \mathrm{fs}$ time step, while quantum equations were integrated with $0.025 \mathrm{fs}$, using interpolated quantities between classical steps. The maximum simulation time was $300 \mathrm{fs}$.

Hopping probabilities between excited states were computed with the fewest switches approach $^{52}$ including decoherence corrections $\left(\alpha=0.1\right.$ Hartree). ${ }^{53}$ Nonadiabatic couplings with 
$\mathrm{ADC}(2)$ were computed by finite differences with the method discussed in Ref. ${ }^{54}$, which is based on the Hammes-Schiffer-Tully approach. ${ }^{55}$ As a single-reference and low-order method, ADC(2) cannot provide reliable nonadiabatic couplings for crossings with the ground state. ${ }^{54}$ For this reason, when a trajectory reached an $\mathrm{S}_{1}-\mathrm{S}_{0}$ energy gap smaller than $0.15 \mathrm{eV}$ before the maximum simulation time, it was stopped. This crossing time was taken as an estimate of the internal conversion time to the ground state.

$\operatorname{ADC}(2)$ calculations were carried out with the Turbomole program. ${ }^{56}$ The spectrum and dynamics simulations were performed with Newton- $\mathrm{X}^{57,58}$ interfaced with Turbomole. Surface intersections (within $0.01 \mathrm{eV}$ ) were optimized with the penalty Lagrange multiplier technique $(\alpha=$ 0.02 hartree) implemented in the Ciopt program, ${ }^{59}$ which we have adapted to work with $\operatorname{ADC}(2)$ and Turbomole. Cremer-Pople parameters were computed with Platon. ${ }^{60}$

\section{Ensemble Sampling}

\section{Quantum sampling}

The ground-state phase space of a molecule with $N_{a t}$ atoms can be conveniently sampled by a harmonic-oscillator Wigner distribution

$$
P_{W}\left(\mathbf{q}, \mathbf{p}_{q}\right)=\frac{1}{(\pi \hbar)^{3 N_{a t}-6}} \prod_{i=1}^{3 N_{a t}-6} \exp \left(\frac{-q_{i}^{2}}{2 \sigma_{q i}^{2}}\right) \exp \left(\frac{-p_{i}^{2}}{2 \sigma_{p i}^{2}}\right)
$$

where

$$
\begin{aligned}
\sigma_{q i}^{2} & =\frac{\hbar}{2 \mu_{i} \omega_{i}} \\
\sigma_{p i}^{2} & =\frac{\hbar \mu_{i} \omega_{i}}{2}
\end{aligned}
$$

In these equations, $q_{i}$ and $p_{i}$ are the coordinate and momentum for each normal mode $i$ with reduced mass $\mu_{i}$ and angular frequency $\omega_{i} . N_{p}=2000$ nuclear geometries and momenta were sampled using Eq. (2) and converted back to Cartesian coordinates. The normal coordinates for the electronic ground state minimum were computed at MP2/(aug)-cc-pVDZ. This set will be referred to as the Q sampling throughout the paper.

\section{Thermal sampling}

A Born-Openheimmer (BO) ground-state dynamics trajectory was run for 10 ps with $1 \mathrm{fs}$ time step using MP2/(aug)-cc-pVDZ. Canonical ensemble (300 K) was enforced via the Andersen thermostat ${ }^{61}$ with $0.2 \mathrm{fs}^{-1}$ collision frequency. A total of 20 snapshots were randomly selected between 5 and $10 \mathrm{ps}$ 
of this trajectory to spawn 20 new trajectories. These trajectories run for 25 ps with the same parameters as the original one. Combined, these new trajectories corresponded to 500 ps ground-state dynamics. $N_{p}=2000$ snapshots (geometries and velocities) were randomly selected from this set and used to compute the absorption spectrum and also to sample the initial conditions for the excited state dynamics. This set will be referred to as the $T_{300}$ sampling. The same procedure was also adopted to build a second thermal ensemble at $T=912 \mathrm{~K}$ ( $\mathrm{T}_{912}$ sampling).

The thermal sampling was also prepared based on molecular mechanics for pyrrole in the gas phase using AMBER 11 suite of programs..$^{62}$ The charges and parameters of pyrrole were taken from Charmm General Force Field for drug like molecules. ${ }^{63,64}$ Pyrrole was first minimized, then slowly heated from 0 to $300 \mathrm{~K}$ for $30 \mathrm{ps}$, and finally equilibrated for $20 \mathrm{~ns}$ at $300 \mathrm{~K}$ using Langevin thermostat with a collision frequency of $1.0 \mathrm{ps}^{-1}$. After heating, the production run of $500 \mathrm{ps}$ was performed without electrostatic cutoffs and using the Andersen thermostat with $0.01 \mathrm{fs}^{-1}$ collision frequency. The production run was done with 1 fs time step for 500 ps. 5000 snapshots where used for analysis of the ensemble, which will be referred to as the $\mathrm{T}_{300}^{\mathrm{MM}}$ sampling.

\section{Spectrum Simulations}

Ensembles computed with quantum and thermal samplings were used to simulate the absorption cross section based on the nuclear ensemble approach. For each of the $N_{P}$ geometries $\left(\mathbf{R}_{l}\right)$ in the ensemble, transition energies $\left(\Delta E_{0 n}\right)$ and oscillator strengths $\left(f_{\text {on }}\right)$ for $N_{f s}=25$ excited states were computed at $\operatorname{ADC}(2) /\left(\right.$ aug)-cc-pVDZ level. The absorption cross section is given by ${ }^{50}$

$$
\sigma(E)=\frac{\pi e^{2} \hbar}{2 m c \varepsilon_{0} E} \sum_{n}^{N_{f s}} \frac{1}{N_{p}} \sum_{l}^{N_{p}} \Delta E_{0 n}\left(\mathbf{R}_{l}\right) f_{0 n}\left(\mathbf{R}_{l}\right) g\left(E-\Delta E_{0 n}\left(\mathbf{R}_{l}\right), \delta\right),
$$

where $E$ is the photon energy of the radiation, $\varepsilon_{0}$ is the vacuum permittivity, $c$ is the speed of light, and $e$ and $m$ are the electron charge and mass. $g$ is a normalized Gaussian line shape

$$
g\left(E-\Delta E_{0 n}, \delta\right)=\frac{1}{\left(2 \pi(\delta / 2)^{2}\right)^{1 / 2}} \exp \left(\frac{-\left(E-\Delta E_{0 n}\right)^{2}}{2(\delta / 2)^{2}}\right)
$$

centered at $\Delta E_{0 n}$ and with width $\delta=0.05 \mathrm{eV}$. 


\section{Results and Discussion}

\section{Sampling characterization}

We start by analyzing the energy and geometry distributions arising from each type of sampling. In total, we produced four ensembles: one from quantum sampling (Q) based on the harmonic-oscillator Wigner distribution, and three from thermal samplings. Among the thermal sampling, two ensembles were built at $300 \mathrm{~K}$ ( $\mathrm{T}_{300}$ from a $\mathrm{BO}$ trajectory and $\mathrm{T}_{300}^{\mathrm{MM}}$ from a MM trajectory) and the last one at $912 \mathrm{~K}$ ( $\mathrm{T}_{912}$ also from a BO trajectory), as explained later.

The distribution of kinetic and total ground-state energies for the quantum (Q) and thermal samplings at $300 \mathrm{~K}\left(\mathrm{~T}_{300}\right.$ and $\left.\mathrm{T}_{300}^{\mathrm{MM}}\right)$ are shown in Figure 2. The difference between $\mathrm{Q}$ and $\mathrm{T}$ samplings is striking. The mean kinetic and total energies in the ground state are much smaller in the $T_{300}$ and $\mathrm{T}_{300}^{\mathrm{MM}}$ samplings than in the $\mathrm{Q}$ sampling. This happens because in the thermal sampling the mean kinetic energy is $3 N_{a t} k T / 2=0.39 \mathrm{eV}$, while it is $E_{Z P} / 2=1.18 \mathrm{eV}$ in the quantum sampling. Apart of a small shift between the distributions, sampling the ensemble either with a $\mathrm{BO}\left(\mathrm{T}_{300}\right)$ or with a MM trajectory $\left(\mathrm{T}_{300}^{\mathrm{MM}}\right)$ leads to equivalent results.

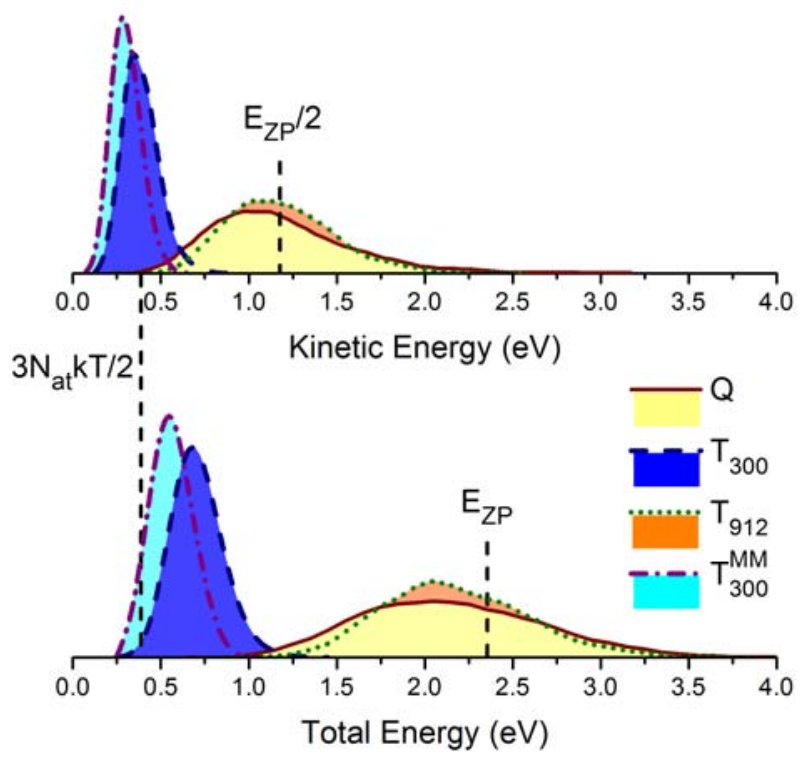

Figure 2. Distribution of ground-state kinetic (top) and total (bottom) energies in the Q and T (BO: $T=$ 300 and $912 \mathrm{~K}$; MM: $T=300 \mathrm{~K}$ ) samplings. $E_{\mathrm{ZP}}=2.36 \mathrm{eV}$ is the harmonic zero-point energy computed at the same QM level.

We may also build the thermal ensemble with a temperature $T_{Z P}$ set to correspond to the zero point energy $E_{Z P}$ : 


$$
\frac{E_{Z P}}{2}=\frac{3}{2} N_{a t} k T_{Z P}
$$

For pyrrole, $T_{Z P}=912 \mathrm{~K}$. In this case, the mean value of the $T_{912}$ kinetic-energy distribution is displaced to $E_{\mathrm{ZP}} / 2$ by construction.

Figure 2 also shows that the width of the energy distributions (kinetic and total) is much narrower in the $\mathrm{T}_{300}$ and $\mathrm{T}_{300}^{\mathrm{MM}}$ samplings than in the $\mathrm{Q}$ sampling. To understand this difference, notice that the total energy variance in the quantum sampling based on a harmonic-oscillator Wigner distribution is

$$
\sigma_{E Q}^{2}=\sum_{i=1}^{3 N_{a t}-6}\left[\left(\frac{\sigma_{p i}^{2}}{2 \mu_{i}}\right)^{2}+\left(\frac{\mu_{i} \omega_{i} \sigma_{q i}^{2}}{2}\right)^{2}\right]=\frac{1}{2} \sum_{i=1}^{3 N_{a t}-6}\left(\hbar \omega_{i}\right)^{2},
$$

yielding a quantum distribution with width $2 \sigma_{E Q}=1.12 \mathrm{eV}$ for pyrrole. In the case of the thermal sampling, supposing that the molecular vibration can be described as Boltzmann-distributed independent harmonic oscillators, the energy variance is

$$
\sigma_{E T}^{2}=\left(3 N_{a t}-6\right) k^{2} T^{2}
$$

Thus, the width of the classical distribution is $2 \sigma_{E T}=0.25 \mathrm{eV}$, much narrower than the width of the quantum distribution. Once more, we may check the performance of the $\mathrm{T}_{912}$ sampling. As shown in Figure 2, the result is in good agreement with the Q sampling.

In the literature, it is also common to sample the geometries using a harmonic oscillator distribution, but then constraining velocity magnitude such that the total energy is the $E_{Z P}$. This procedure, used for instance in Ref. ${ }^{40}$, results in a total energy distribution for the quantum sampling with zero variance.

In terms of coordinates, the deviations between the distributions of the T- and Q-samplings depend on the specific vibrational frequencies, as expressed in Eq. (1) (see also Figure 1-bottom). In Figure 3, we plot the distributions of three internal coordinates in the ground state for the thermal and quantum samplings.

The first internal coordinate (Figure 3-top) is the NH distance. In the Q sampling, the main normal mode contributing to this coordinate is the NH stretching vibrating at $3683 \mathrm{~cm}^{-1}$. As expected (Eq. (1)), this distribution is much wider than that obtained for both $\mathrm{T}$ samplings at $300 \mathrm{~K}$. The deviations between $T_{300}$ and $\mathrm{Q}$ are much smaller for the $\mathrm{CN}$-distance distributions $\left(\sim 1200 \mathrm{~cm}^{-1}\right.$, see Figure 3-middle), and almost negligible for the CNCC-dihedral distributions $\left(\sim 650 \mathrm{~cm}^{-1}\right)$. 


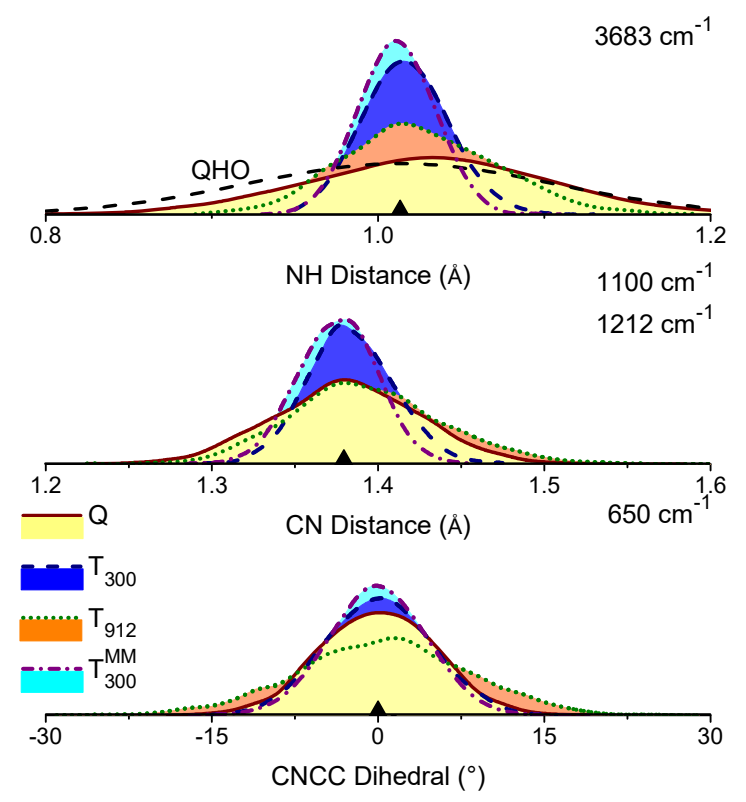

Figure 3. Distributions of $\mathrm{NH}$ distances, $\mathrm{CN}$ distances, and $\mathrm{CNCC}$ dihedral angles for the quantum and thermal (BO: $T=300$ and $912 \mathrm{~K}$; MM: $T=300 \mathrm{~K}$ ) samplings. The ground-state harmonic oscillator wave function for the N-H stretching mode is indicated the upper graph as well (QHO). The triangles indicate equilibrium values. Wavenumbers of the main modes contributing to the variation of each coordinate are also shown. The areas of all graphs are normalized to one.

The overheated $\mathrm{T}_{912}$ sampling produces distributions that are equivalent to those of the $\mathrm{Q}$ sampling for middle-range wavenumbers, like in the case of the $\mathrm{CN}$-distance distribution (Figure 3middle). However, it produces too narrow distributions for high-frequency cases (Figure 3-top) and too broad distributions for low-frequency cases (Figure 3-bottom). We will see in the next section that this unbalanced distribution of energy among the modes leads to artifacts in the spectrum simulations.

Once more, we observe that there is no significant difference between using a $\mathrm{BO}$ or MM trajectories to sample the geometries in $\mathrm{T}_{300}$ and $\mathrm{T}_{300}^{\mathrm{MM}}$. For this reason, in the next section we will proceed only with $\mathrm{BO}$ samplings, $\mathrm{T}_{300}$ and $\mathrm{T}_{912}$, to simulate the spectrum.

\section{Absorption Spectrum}

We have used the nuclear ensemble approach to simulate the absorption spectrum of pyrrole based on $A D C(2)$ excitation energies and oscillator strengths for the $\mathrm{Q}, \mathrm{T}_{300}$, and $\mathrm{T}_{912}$ samplings.

The nuclear ensemble approach is possibly the simplest approach to simulate electronic spectrum including band shapes. In this method, energies and transition probabilities (proportional to the oscillator strengths) are computed for each geometry in an ensemble representing the initial state distribution, and then summed over (see Eq. (4)) to provide the spectrum. ${ }^{50,65}$ The nuclear ensemble 
approach is intuitive, straightforward to implement, and delivers absolute widths and heights even for dark vibronic bands. However, as it does not incorporate any information on the final states wave functions, it completely misses vibrationally resolved features, and tends to predict too symmetric band shapes. (This and more involved approaches for spectrum simulations are discussed, for instance, in Ref. ${ }^{66}$.) The nuclear ensemble approach is also very handy as the initial step for excitedstate dynamics simulations. The same set of geometries used for the spectrum, together with their corresponding momentum, can be used as the initial conditions to start semiclassical trajectories.

The vertical excitations of pyrrole computed with $\mathrm{ADC}(2)$ are shown in Table 1 . The rootmean-square deviation (RMSD) to the vertical energies computed with CC3 (Ref. ${ }^{10}$ ) is $0.2 \mathrm{eV}$. Most of vertical transitions are slightly overestimated compared to $\mathrm{CC} 3$, with exception of the $1{ }^{1} \mathrm{~A}_{1}$ state, the only state in the series with a dominant valence character. The triple- $\zeta$ basis marginally stabilizes the double- $\zeta$ results by $0.1 \mathrm{eV}$ (RMSD).

Table 1 - Singlet vertical excitations of pyrrole at ADC(2)/(aug)-cc-pVDZ. Velocity-gauge oscillator strengths. The experimental ${ }^{25}$ oscillator strength between 5.5 and $6.5 \mathrm{eV}$ is given in the parenthesis. Results at ADC(2)/aug-cc-pVTZ are given within brackets.

\begin{tabular}{|c|c|c|c|c|c|}
\hline \multirow[b]{2}{*}{ State } & \multicolumn{2}{|l|}{$\mathrm{ADC}(2)$} & \multirow{2}{*}{$\frac{C C 3^{10}}{\Delta E(\mathrm{eV})}$} & \multirow[b]{2}{*}{ Assignment } & \multirow[b]{2}{*}{ Expt. $(\mathrm{eV})^{25}$} \\
\hline & $\Delta E(\mathrm{eV})$ & $f$ & & & \\
\hline $1^{1} \mathrm{~A}_{2}$ & $5.27[5.22]$ & 0.000 & 5.10 & $\pi \rightarrow 3 \mathrm{~s}$ & \\
\hline $1^{1} \mathrm{~B}_{1}$ & $5.94[5.91]$ & 0.006 & 5.85 & $\pi \rightarrow 3 \mathrm{p}_{\mathrm{yz}}$ & \\
\hline $2^{1} \mathrm{~B}_{1}$ & $6.04[6.01]$ & 0.034 & 5.99 & $\pi \rightarrow 3 \mathrm{~s}$ & $5.861^{a}$ \\
\hline $2^{1} \mathrm{~A}_{2}$ & $6.11[5.96]$ & 0.000 & 5.86 & $\pi \rightarrow 3 \mathrm{~s}$ & \\
\hline $1^{1} \mathrm{~B}_{2}$ & $6.25[6.25]$ & 0.143 & 5.98 & $\pi \rightarrow 3 \mathrm{p}_{\mathrm{x}}$ & $6.12^{b}(0.12)$ \\
\hline $2^{1} \mathrm{~A}_{1}$ & $6.33[6.30]$ & 0.001 & 6.37 & $\pi \rightarrow \pi^{*} / 3 \mathrm{p}_{\mathrm{x}}$ & \\
\hline $3{ }^{1} \mathrm{~B}_{1}$ & $6.58[6.55]$ & 0.012 & 6.47 & $\pi \rightarrow 3 \mathrm{~d}_{\mathrm{yz}}$ & \\
\hline $3^{1} \mathrm{~A}_{2}$ & $6.60[6.53]$ & 0.000 & 6.43 & $\pi \rightarrow 3 \mathrm{~d}_{\mathrm{yz}}$ & \\
\hline $4^{1} \mathrm{~B}_{1}$ & $6.82[6.71]$ & 0.024 & 6.72 & $\pi \rightarrow 3 \mathrm{p}_{\mathrm{yz}}$ & \\
\hline $4^{1} \mathrm{~A}_{2}$ & $6.85[6.79]$ & 0.000 & 6.50 & $\pi \rightarrow 3 \mathrm{~d}_{\mathrm{yz}}$ & \\
\hline $2^{1} \mathrm{~B}_{2}$ & $7.02[6.82]$ & 0.002 & 6.63 & $\pi \rightarrow 3 \mathrm{p}_{\mathrm{x}} / \pi^{*}$ & \\
\hline
\end{tabular}

${ }^{a}$ Origin of the state. ${ }^{b}$ Estimated "experimental vertical excitation" (see text); its origin is at $5.698 \mathrm{eV}$ and the band maximum is at $5.95 \mathrm{eV}$.

The experimental spectrum ${ }^{25}$ of pyrrole below $6.5 \mathrm{eV}$ is dominated by a broad and intense transition into the $1{ }^{1} \mathrm{~B}_{2}$ state, which is peaked at $5.95 \mathrm{eV}$ (Figure 4). A narrow intense peak due to the origin of the $2{ }^{1} \mathrm{~B}_{1}$ transition features at $5.86 \mathrm{eV}$. A recent assignment of pyrrole spectrum based on quantum-dynamics simulations is discussed in Ref. $^{33}$.

The band maximum is usually taken as the reference value to check computed vertical excitations. Nevertheless, to properly compare theory and experiment, especially in the case of a broad band as that of the $1^{1} \mathrm{~B}_{2}$ transition, we should take into account that the experimental band maximum does not correspond to the vertical excitation. We can estimate the deviation between the band maximum and the vertical excitation from the simulated spectrum. The simulated band maximum (Q sampling, Figure 4) is at $6.08 \mathrm{eV}$. The vertical excitation into the $1^{1} \mathrm{~B}_{2}$ state is at $6.25 \mathrm{eV}$ 
(Table 1), $0.17 \mathrm{eV}$ higher than the maximum. Assuming that the same shift applies to the experimental data, whose band maximum is at $5.95 \mathrm{eV}$, the "experimental vertical excitation" may be taken as $6.12 \mathrm{eV}$. The agreement between the $\mathrm{ADC}(2)$ vertical excitation into the $1^{1} \mathrm{~B}_{2}$ state $(6.25 \mathrm{eV}$ for both basis sets) and the "experimental vertical excitation" $(6.12 \mathrm{eV})$ is excellent.

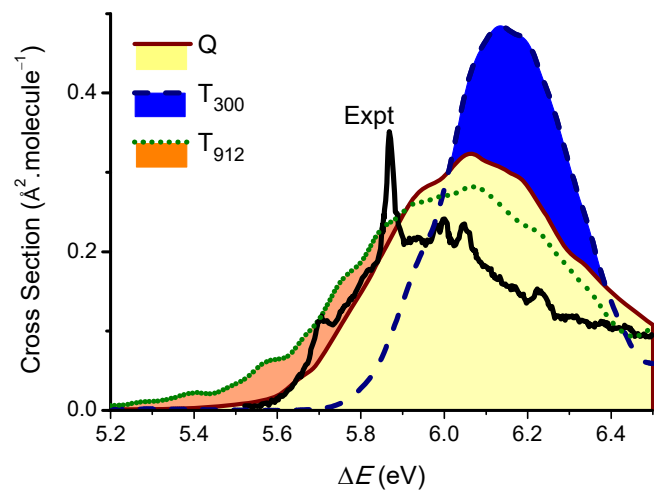

Figure 4. Photoabsorption cross section computed with the quantum and thermal ( $T=300$ and $912 \mathrm{~K})$ samplings compared to the experimental results. ${ }^{25}$

The absorption spectrum computed with the thermal and quantum samplings based on $\operatorname{ADC}(2) /\left(\right.$ aug)-cc-pVDZ states are shown in Figure 4. The experimental result from Ref. ${ }^{25}$ is also shown in the figure. At $300 \mathrm{~K}$, the $\mathrm{T}_{300}$ sampling produces a spectrum that has qualitatively wrong band shape, when compared to the experimental result. The spectrum based on the $\mathrm{Q}$ sampling provides a much better result for the band shape, especially in the low-energy edge.

The failure of the $\mathrm{T}_{300}$ sampling in the description of the low-energy edge is related to the geometry of the bright $\pi \pi^{*}$ minimum. This minimum, the $\mathrm{S}_{4}$ state with $\mathrm{ADC}(2)$, is reached by shortening the $\mathrm{C} 3-\mathrm{C} 4$ bond (by $0.06 \AA$ at $\mathrm{ADC}(2)$ level) and stretching the $\mathrm{C} 2-\mathrm{C} 3$ and $\mathrm{C} 5-\mathrm{C} 4$ bonds (both by $0.07 \AA$ ). These bond deformations, which lead to small energy gaps of about $5.7 \mathrm{eV}$, are not well described by the $\mathrm{T}_{300}$ sampling.

The spectrum computed with the $\mathrm{T}_{912}$ sampling is also shown in Figure 4 . The result is significantly better than at $300 \mathrm{~K}$, with the band shape much closer to the experimental one. There is, however, an overestimation of the absorption cross section in the low-energy edge. This is caused by the excess of energy deposited in ring-deformation modes, which leads to artificially too broad distributions as shown in Figure 3-bottom.

\section{Dynamics simulations}

To simulate semiclassical dynamics, we need an ensemble of initial geometries and velocities to start each trajectory. Rigorously, surface hopping should be based on a double ensemble strategy: ${ }^{51}$ 
trajectories are started from different geometries and velocities representing the initial distribution of the molecule in the phase space; and for each of these points several trajectories should also be started to sample the random nonadiabatic processes. (Different from classical dynamics, in surface hopping, trajectories initiated with the same conditions may have different fates due to the stochastic nature of the hops.) Commonly, to reduce the computational costs, this double-ensemble approach is replaced by a single ensemble, where only a single trajectory is started from each phase-space point. This is the approach we adopt here as well.

Two sets of excited-state dynamics simulations were run, one starting from initial conditions generated with the $\mathrm{Q}$ sampling and another with the $\mathrm{T}_{300}$ sampling. In both cases, initial conditions were restricted to the $6.1 \pm 0.1 \mathrm{eV}$ energy window, exciting the $\pi \rightarrow 3 \mathrm{p}_{\mathrm{x}}$ state near the center of the first absorption band. 100 trajectories were computed for each sampling. The number of trajectories starting in each adiabatic state (see Table 2) was taken as proportional to the transition probabilities into each state within the energy window. Thus, in the Q sampling, for instance, 19 trajectories were started in $\mathrm{S}_{3}, 30$ in $\mathrm{S}_{4}, 36$ in $\mathrm{S}_{5}$, and 15 in $\mathrm{S}_{6}$. This procedure ensures that the initial conditions in both samplings are equivalent in terms of excitation energy and diabatic character of the initial state.

Table 2 - Distribution of ensemble points among the electronic states according to their oscillator strengths, in the $6.1 \pm 0.1 \mathrm{eV}$ energy window. The number of initial conditions (IC) was taken approximately proportional to the nuclear ensemble (NE) up to a total of 100 initial conditions.

\begin{tabular}{|llllllllll|}
\hline & Sampling & $\mathrm{S}_{1}$ & $\mathrm{~S}_{2}$ & $\mathrm{~S}_{3}$ & $\mathrm{~S}_{4}$ & $\mathrm{~S}_{5}$ & $\mathrm{~S}_{6}$ & $\mathrm{~S}_{7}$ & $\mathrm{~S}_{7}-\mathrm{S}_{25}$ \\
\hline $\mathrm{NE}$ & $\mathrm{Q}$ & 0 & 18 & 120 & 218 & 262 & 112 & 2 & 0 \\
$\mathrm{NE}$ & $\mathrm{T}_{300}$ & 0 & 13 & 246 & 286 & 408 & 126 & 0 & 0 \\
$\mathrm{NE}$ & $\mathrm{T}_{912}$ & 0 & 19 & 108 & 192 & 208 & 118 & 1 & 0 \\
$\mathrm{IC}$ & $\mathrm{Q}$ & 0 & 0 & 19 & 30 & 36 & 15 & 0 & 0 \\
$\mathrm{IC}$ & $\mathrm{T}_{300}$ & 0 & 0 & 23 & 27 & 38 & 12 & 0 & 0 \\
\hline
\end{tabular}

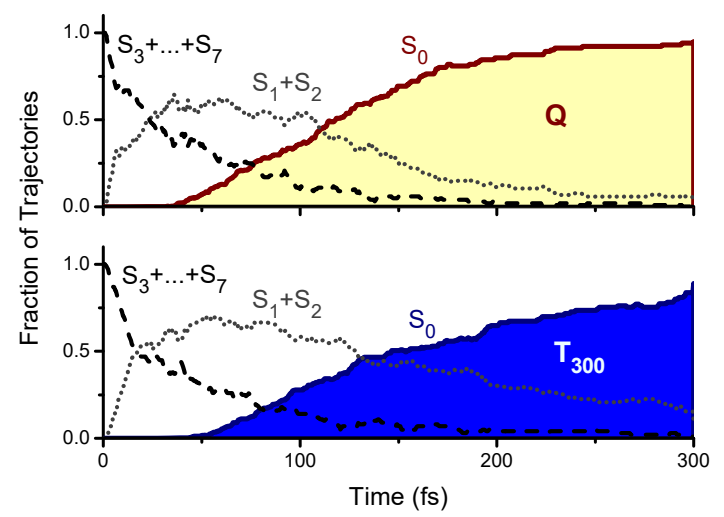

Figure 5. Fraction of trajectories as a function of time in $\mathrm{S}_{0}, \mathrm{~S}_{1}+\mathrm{S}_{2}$, and in the $\mathrm{S}_{3}+\ldots+\mathrm{S}_{7}$ groups of states for the $\mathrm{Q}$ and $\mathrm{T}_{300}$ samplings. 
The general time-evolution of the trajectories is illustrated in Figure 5. Qualitatively, the results for $Q$ and $T_{300}$ samplings are similar. The occupation of the initially populated states $\left(S_{3}\right.$ to $\left.S_{6}\right)$ quickly reduces; first populating $\mathrm{S}_{2}$ and $\mathrm{S}_{1}$ with time constant $\tau_{1}$ and then $\mathrm{S}_{0}$ with $\tau_{0}$ :

$$
\left[S_{3}+\ldots+S_{7}\right] \stackrel{1 / \tau_{12}}{\longrightarrow}\left[S_{1}+S_{2}\right] \stackrel{1 / \tau_{0}}{\longrightarrow}\left[S_{0}\right]
$$

With such a partition (which includes $S_{7}$ in the first group to account for the up-hops from $S_{6}$ ), we can fit the $\left[\mathrm{S}_{1}+\mathrm{S}_{2}\right]$ occupation analogously to a unimolecular reaction with an intermediary step

$$
F_{S 1+S 2}(t)=\frac{\tau_{0}}{\tau_{12}-\tau_{0}}\left[\exp \left(-\frac{t}{\tau_{12}}\right)-\exp \left(-\frac{t}{\tau_{0}}\right)\right] .
$$

The result of this fitting is given in Table 3. Clearly, the initial transfer into $S_{2}$ and $S_{1}$ is independent of the sampling, and about 27 fs. Nevertheless, the transfer from $S_{2}$ and $S_{1}$ into $S_{0}$ with $Q$ sampling is significantly faster than that with the $\mathrm{T}_{300}$ sampling. While with the $\mathrm{Q}$ sampling $\mathrm{S}_{0}$ is populated within approximately $\tau_{0}=90 \mathrm{fs}$, with the $\mathrm{T}_{300}$ sampling it takes about $148 \mathrm{fs}$.

Table 3 - Characterization of the T-sampled $(300 \mathrm{~K})$ and Q-sampled dynamics in the $6.1 \pm 0.1 \mathrm{eV}$ excitation window. Time constants: $\tau_{12}$ for exponential transfer from the high excited states into $S_{1}$ and $S_{2} ; \tau_{1}$ for the $S_{2}$ transfer to $S_{1} ; \tau_{0}$ for the transfer from $S_{1}$ to $S_{0} ; \tau_{H}$ for $S_{1}$ to $S_{0}$ transfer via $H$ elimination; $\tau_{R}$ for $S_{1}$ to $S_{0}$ transfer via ring distortion; $\tau_{\pi \sigma^{*}}$ for crossing from $\pi 3 \mathrm{~s}$ to $\pi \sigma^{*}$ (H fragments formation).

\begin{tabular}{|lcc|}
\hline & Sampling \\
\cline { 2 - 3 } Time constant $(\mathrm{fs})$ & $\mathrm{Q}$ & $\mathrm{T}_{300}$ \\
\hline$\tau_{12}$ & 27 & 28 \\
$\tau_{1}$ & 55 & 89 \\
$\tau_{0}$ & 90 & 148 \\
$\tau_{\mathrm{H}}$ & 87 & 154 \\
$\tau_{\mathrm{R}}$ & 98 & 124 \\
$\tau_{\pi \sigma^{*}}$ & 64 & 100 \\
\hline
\end{tabular}

There is also an important difference in the activation of the different reaction paths. Pyrrole internal conversion may occur either through a $\pi \sigma^{*} /$ ground-sate intersection induced by the $\mathrm{NH}$ stretching (H-elimination pathway) or through $\pi \pi * /$ ground-state intersection induced by simultaneous ring opening and puckering (ring-distortion pathway). ${ }^{37}$ Dynamics starting from the $\mathrm{Q}$ sampling features $59 \%$ of $\mathrm{H}$-elimination, against $70 \%$ when starting from the $\mathrm{T}_{300}$ sampling (Figure 6-top). For 100 trajectories, the uncertainty in these values is $\pm 8 \%$ for $90 \%$ confidence interval. Thus, in spite of some overlap in the extreme of the error bars, it seems that the $T_{300}$ sampling really leads to a preference for $\mathrm{H}$ elimination in comparison to the $\mathrm{Q}$ sampling.

There is an extensive patch of ring-distortion crossing seam that can give rise to internal conversion to the ground state. ${ }^{67}$ It varies in terms of degree and type of ring puckering. The distribution of ring-distortions at the $\mathrm{S}_{1} / \mathrm{S}_{0}$ crossing point given by the Cremer-Pople parameters ${ }^{68} \varphi$ and $\mathrm{Q}$ are shown in Figure 6-bottom. For both samplings, internal conversion to the ground state tends 
to occur with a substantial puckering degree, between 0.3 and $0.5 \AA$. Also for both, there is a large variation of types of puckering specially from envelope conformations involving the $\mathrm{N}$ atom $\left(\mathrm{E}_{1}\right)$ to twisted conformations involving $\mathrm{C} 2$ and $\mathrm{C} 3\left({ }^{2} \mathrm{~T}_{3}\right)$. It is not possible, however, to distinguish any special trends in either sampling.
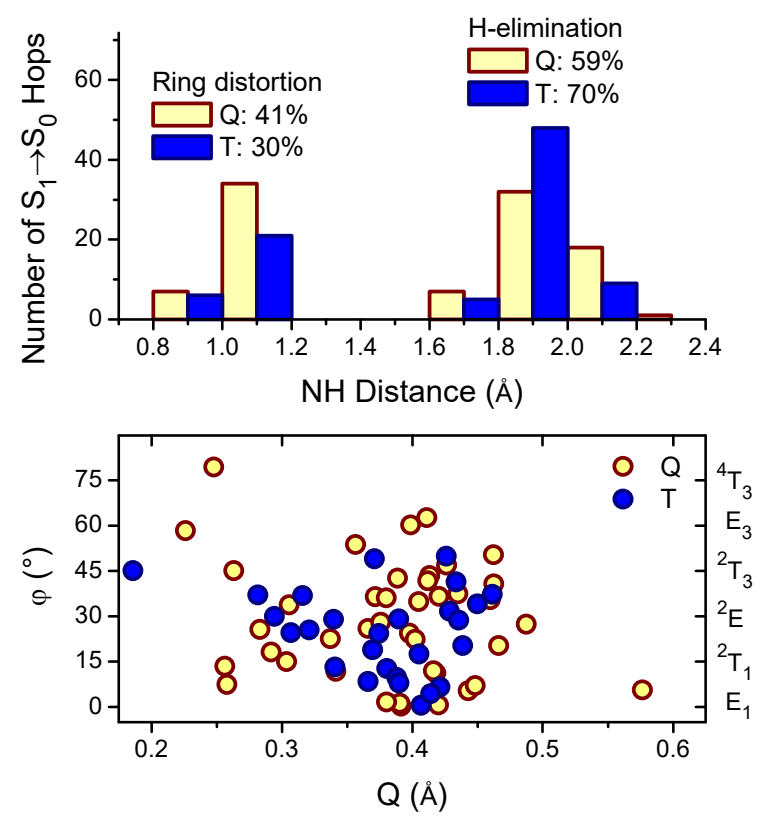

Figure 6. Top: Number of $\mathrm{S}_{1} \rightarrow \mathrm{S}_{0}$ hops as a function of the NH distance. For $90 \%$ confidence interval, the errors in the fractions of ring distortion and H-elimination are $\pm 8 \%$. Bottom: Cremer-Pople parameters at the $\mathrm{S}_{1} \rightarrow \mathrm{S}_{0}$ hop time for the ring-distortion cases. The corresponding puckering conformations are indicated at the right axis. $\mathrm{E}_{n}$ : envelope puckering with atom $n$ below the ring plane (in ${ }^{n} \mathrm{E}, n$ is above the plane); ${ }^{m} \mathrm{~T}_{n}$ : twisted puckering with $m$ above and $n$ below the plane. $\mathrm{C} 2 \leftrightarrow \mathrm{C} 5$ and $\mathrm{C} 3 \leftrightarrow \mathrm{C} 4$ equivalence was taken into account, such as that, for instance, $\mathrm{E}_{5}$ is counted as $\mathrm{E}_{2}$.

The bias of $\mathrm{T}_{300}$ towards $\mathrm{H}$ elimination is somewhat counterintuitive. We could expect that with a narrower $\mathrm{NH}$-distance distribution than in the $\mathrm{Q}$ sampling, the activation of the $\mathrm{H}$ elimination would also reduce. The reason for having the opposite output is related to the nonadiabatic events bringing pyrrole from $S_{2}$ to $S_{1}$. If we apply Eq. (10) to fit the $S_{1}$ occupation instead of $S_{1}+S_{2}$, we see that the $\mathrm{S}_{2} \rightarrow \mathrm{S}_{1}$ transfer time is $\tau_{1}=55 \mathrm{fs}$ in the $\mathrm{Q}$ and $89 \mathrm{fs}$ in the $\mathrm{T}_{300}$ sampling. This reflects the smaller momentum of $T_{300}$ trajectories when they reach the $S_{2} / S_{1}$ crossing seam, compared to the momentum of $\mathrm{Q}$ trajectories in the same region. The Landau-Zener model ${ }^{69}$ of nonadiabatic transitions helps to understand what happens then: in a two-state crossing, everything else being the same, the larger the momentum, the larger the probability of remaining in the same diabatic pathway. In this way, as the $S_{2} / S_{1}$ crossing seam is reached along ring-distortion modes, ${ }^{37}$ trajectories with larger momentum are more likely to remain moving along the ring distortion pathway than those with 
smaller momentum, which have larger probabilities of changing to another diabatic pathway, resulting in H-elimination.

With the exception of the time constant for transfer the populations from high excited states into $S_{1}$ and $S_{2}\left(\tau_{12}\right)$, dynamics is clearly faster with $Q$ than with $T_{300}$ sampling (see Table 3 ). The $\mathrm{S}_{1} \rightarrow \mathrm{S}_{0}$ time constant for trajectories following the ring-distortion path is $\tau_{\mathrm{R}}=98 \mathrm{fs}$ with $\mathrm{Q}$ and somewhat slower with $\mathrm{T}_{300}, 124$ fs. For $\mathrm{H}$ elimination, this time increases from $\tau_{\mathrm{H}}=87$ to $154 \mathrm{fs}$ between the $\mathrm{Q}$ and the $\mathrm{T}_{300}$ samplings.

\section{Comparison to experimental data}

We can summarize the dynamics of pyrrole excited into the $\pi \pi^{*}$ band as schematically illustrated in Figure 7. This figure shows the singlet (neutral) and doublet (cation) states of pyrrole along the Helimination and ring distortion computed by linear interpolation of internal coordinates between the ground state minimum and the optimized $S_{1} / S_{0}$ intersections of each path. These calculations were done at the same level as the spectrum and dynamics simulations, ADC(2)/(aug)-cc-pVDZ. In the following description, we will only mention the Q-sampling time constants and populations fractions. Qualitatively, the $\mathrm{T}_{300}$ description is exactly the same, but with the values provided in Table 2, Table 3, and Figure 6.

Excitation into the $6.1 \pm 0.1 \mathrm{eV}$ (Figure 7a) populates the $\mathrm{S}_{3}-\mathrm{S}_{6}$ manifold, especially $\mathrm{S}_{4}$ and $\mathrm{S}_{5}$. After that, pyrrole quickly relaxes to the $S_{2}$ state in only 27 fs. $S_{1}$ is populated through a $S_{2} / S_{1}$ crossing induced by out-of-plane ring modes (Figure 7b) within 55 fs. The split between the two deactivation paths happens at this crossing: part of the population continues moving along the ring distortion, and the remaining population planarizes and starts the $\mathrm{H}$ elimination. The population following the ring distortion $\left(41 \pm 8 \%\right.$ ) reaches the $\mathrm{S}_{1} / \mathrm{S}_{0}$ intersection (Figure $7 \mathrm{c}$ ) within $98 \mathrm{fs}$. The population following H-elimination (59\%) reaches the $\mathrm{S}_{1} / \mathrm{S}_{0}$ intersection (Figure $7 \mathrm{~d}$ ) within $87 \mathrm{fs}$. Both paths considered, pyrrole returns to the ground state within $90 \mathrm{fs}$.

The split of population between the two pathways has been systematically neglected in diverse experimental works, which usually take into account only the $\mathrm{H}$ elimination. However, photodissociation experiments at $193 \mathrm{~nm}(6.42 \mathrm{eV})^{15}$ showed that pyrrole splits half-half between $\mathrm{C}_{4} \mathrm{NH}_{4}+\mathrm{H}$ and $\mathrm{C}_{3} \mathrm{H}_{4} \mathrm{~N}+\mathrm{HCN}$ channels. Moreover, the $\mathrm{H}$ fragments also split between fast and slow fractions, corresponding to two different mechanisms. Fast $\mathrm{H}$ fragments arise from the H-elimination pathway, while slow $\mathrm{H}$ fragments arise from statistical dissociation in the hot ground state competing with $\mathrm{HCN}$ production. ${ }^{24}$ Therefore, the measured systematic increase of slow $\mathrm{H}$ fragments between 254 and $193.3 \mathrm{~nm}^{24}$ is an indication that another relaxation path but $\mathrm{H}$-elimination is becoming more and more important at higher excitation energies. The only known alternative for this relaxation pathway is the ring distortion. 


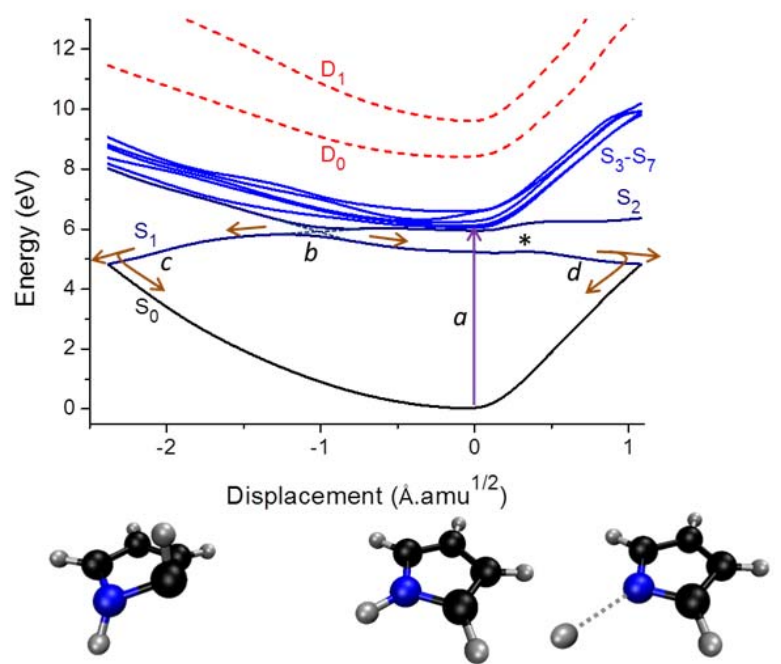

Figure 7. Potential energy profiles of pyrrole along the H-elimination (positive coordinates) and ringdistortion (negative) paths. The ground and singlet excited states (up to $\mathrm{S}_{7}$ ) are shown along with the cation (doublet) states $\mathrm{D}_{0}$ and $\mathrm{D}_{1}$. The geometry at left corresponds to the ring-distorted $\mathrm{S}_{1} / \mathrm{S}_{0}$ intersection; at the center (displacement zero) is the minimum of the ground state; at right is the H-elimination $\mathrm{S}_{1} / \mathrm{S}_{0}$ intersection. The * indicates the top of the $\mathrm{S}_{1}$ barrier.

$\mathrm{HCN}$ and slow $\mathrm{H}$ fragments should result from deactivation through ring-distorted pathways. For this reason, we expect that the ring-distortion/H-elimination branching in the dynamics simulations of pyrrole excited into the $\pi \pi^{*}$ band should also be large, about $50 \%$ to cope with the experimental results. The results from Q sampling (41\% ring distortion against 59\% $\mathrm{H}$ elimination; see Figure 6-top) are doing better in this analysis than those from $\mathrm{T}_{300}$ (30\% ring distortion). In any case, either with $\mathrm{Q}$ or $\mathrm{T}_{300}$ samplings, the substantial split between the two reaction paths is a very positive point for ADC(2). Previous full dimensional dynamics of pyrrole carried out with MRCI and TDDFT failed to show such split. ${ }^{36,38}$

The comparison between experiment and theory concerning the time to reach the ground state poses more challenges. The experiments usually are based on transient variation of the ionization potential (IP) or H-fragments formation. In both, what is measured is not exactly the internal conversion to the ground state. In the first case, the IP strongly varies along the reaction coordinates, a feature that, although recognized, ${ }^{19}$ has not been taken into account yet. As we can see in Figure 7, while in the Franck-Condon region (displacement zero), ionization from $\mathrm{S}_{1}$ into $\mathrm{D}_{0}$ requires $4.29 \mathrm{eV}$ $(289 \mathrm{~nm})$, in the ring-distorted $\mathrm{S}_{1} / \mathrm{S}_{0}$ intersection it requires $9.05 \mathrm{eV}(137 \mathrm{~nm})$ and in the H-elimination $\mathrm{S}_{1} / \mathrm{S}_{0}$ intersection it requires $9.81 \mathrm{eV}(126 \mathrm{~nm})$. In Ref. ${ }^{16}$, up to $3 \times 800 \mathrm{~nm}(4.65 \mathrm{eV})$ probe pulses were used to ionize pyrrole, while in Ref. ${ }^{1}$, the probe pulse was fixed at $266 \mathrm{~nm}(4.66 \mathrm{eV})$. In both cases, after the excitation, pyrrole should leave the ionization window much before reaching the intersection to the ground state, rendering time constants that should be shorter than the $\mathrm{S}_{1} / \mathrm{S}_{0}$ internal conversion 
time. In fact, these experiments may be only probing the time for pyrrole to leave the Franck-Condon region.

In the case of H-fragment detection, the first problem is that such experimental setup neglects the fraction of the population deactivating through ring-distortion. Second, because these experiments are based on $1 \mathrm{~s} \rightarrow 2 \mathrm{~s}$ excitation of $\mathrm{H}$ atoms followed by its resonant ionization, ${ }^{17,21}$ they may detect $\mathrm{H}^{+}$ fragments arising from semi-dissociated pyrrole still in the excited state, leading to an underestimation of the lifetime. This happens because the atomic $2 \mathrm{~s}$ state correlates with the $\pi \sigma^{*}$ state of pyrrole, which becomes the $\mathrm{S}_{1}$ after the $\mathrm{S}_{1}$-barrier along the H-elimination path (the barrier is indicated by a $*$ in Figure 7; before the barrier, $\mathrm{S}_{1}$ is $\pi 3 \mathrm{~s}$ ). Therefore, when the $1 \mathrm{~s} \rightarrow 2 \mathrm{~s}$ excitation takes place, pyrrole may still be in the $\mathrm{S}_{1}$ state. In Ref. ${ }^{17}$, the time constant associated to $\mathrm{H}^{+}$detection after $200 \mathrm{~nm}(6.20 \mathrm{eV})$ excitation of pyrrole is found to be $\tau_{\mathrm{f}}=52 \pm 12$ fs. How may we compare the simulations to this result?

Sapunar and co-workers, ${ }^{35}$ based on surface hopping dynamics also carried out with $\operatorname{ADC}(2)$, directly compared the $\mathrm{S}_{1} / \mathrm{S}_{0}$ deactivation time (in their case, $150 \mathrm{fs}$ ) to $\tau_{\mathrm{f}}$ from Ref. ${ }^{17}$. They attributed the difference between these two values to deficiencies of $\operatorname{ADC}(2)$ to describe the potential energy surface of pyrrole. However, since $\mathrm{H}$ fragments may arise from excited pyrrole, this comparison is not the most adequate.

What should be compared to the experiment is the time that trajectories following the $\mathrm{H}$ elimination path cross from $\pi 3 s$ to $\pi \sigma^{*}$ state. This crossing (indicated by * in Figure 7 ) happens at the $\mathrm{NH}$ distance of $1.29 \AA$. The computational quantity that holds the best equivalence to the quantity measured is the cumulative probability of the trajectories to have reached this threshold distance. Fitting this probability with an exponential model similar to that used to fit the transient spectra in the experiments (see supporting information of Ref. ${ }^{17}$ ) results in a H-fragment formation time of $\tau_{\pi \sigma^{*}}=64$ fs for Q sampling and $100 \mathrm{fs}$ for $\mathrm{T}_{300}$ sampling (Table 3). Compared to the $\tau_{\mathrm{f}}=52 \pm 12 \mathrm{fs}$ experimental value, ${ }^{17}$ these results mean, first, that there is nothing in principle wrong with $\mathrm{ADC}(2)$ simulations and, second, that $\mathrm{Q}$ sampling performs better than $\mathrm{T}_{300}$.

\section{Choosing between quantum and thermal samplings}

Along this paper, we have shown that the results for excited-state dynamics and the absorption spectrum simulations for pyrrole are very dependent on the method chosen for sampling the groundstate geometry distribution. Unsurprisingly, thermal samplings delivered narrower spectrum and slower dynamics than quantum sampling. Although we have studied a single molecule in this work, most probably these are general trends that should be observed for most of molecular systems investigated with similar methodologies. 
We should admit that even before any simulation, we already favored the quantum sampling, as it should be the best representation of a quantum density projected on the classical phase space. The comparison between the simulations and the experiments reinforced this view, as the Q sampling performed clearly better than the $\mathrm{T}$ sampling. Thus, our general prescription is that the $\mathrm{Q}$ sampling should be the preferred method to sample initial conditions for dynamics and spectrum.

We are aware, however, that for large molecular systems, the Q sampling may be computationally too expensive. As applied here (see Eq.(2)) - which could be considered the simplest approximation for a Q sampling - normal displacements are required. To get them may simply be prohibitive, for instance, for typical chromophore-protein systems. One possibility is to resort to a hybrid method like that discussed in Ref. ${ }^{44}$, where the Q sampling is applied only to the chromophore, and the remaining system is thermalized around each frozen chromophore geometry given by the $\mathrm{Q}$ ensemble. With such a method, only normal modes for the chromophore are needed.

A special situation may occur for systems with a large number of conformational or isomerization minima. In such a case, the ensemble and the derived quantities (spectrum and initial conditions) should be weighted by the corresponding Boltzmann factor of each minimum. Again, this may make the application of the $\mathrm{Q}$ sampling prohibitive because one needs to compute normal modes for each minimum. We have faced such a type of system before, studying the spectrum of urocanic acid. ${ }^{70}$ This molecule has diverse tautomers and the final spectral shape was determined by few of them. To deal with a system with multiple minima, we may once more resort to a hybrid methodology, where a thermal sampling is initially used to locate the minima contributing with the largest Boltzmann factors, and then applying the Q to each of them.

Here, we have discussed a sub-picosecond process. However, for much longer processes (tens of picoseconds), the Q sampling may also pose other challenges. First, because of the faster dynamics than with the $\mathrm{T}$ sampling, the quality of the numerical integration of the Newton equations will degrade faster with the Q sampling, which may require shorter time steps to keep the same level of energy conservation than with $\mathrm{T}$ sampling. ${ }^{40}$ Moreover, the initial distribution of energy (zero-point energy in each mode) will tend to leak from fast to slow modes, yielding a distribution similar to that discussed for $\mathrm{T}_{912}$ sampling. Such situation may additionally require the usage of zero-point energy constraints. $^{71}$

\section{Conclusions}

Absorption spectrum and excited-state nonadiabatic dynamics simulations for pyrrole in the gas phase, initiated from thermal (T) and quantum $(\mathrm{Q})$ samplings, show that: 
- The ground-state properties of the ensemble (energies and internal coordinates) are strongly dependent on the sampling method. By construction, Q sampling renders mean energies near the zero-point level, while $\mathrm{T}$ sampling renders much smaller mean energies, near a thermal distribution. The width of the energy distribution is also strikingly different, much broader with Q than with T sampling.

- The difference between the distribution of internal coordinates in the T and Q sampling depends on the normal frequencies contributing to the coordinate. The larger the frequencies are, the larger the differences.

- Overheating the T sampling until it reaches the zero-point level produces good ground-state energy distributions, but creates artifacts in the internal coordinate distributions.

- For all tested properties, T samplings prepared with either Born-Oppenheimer or molecular mechanics produce similar results.

- The absorption spectrum is strongly dependent on the sampling method. T sampling at $300 \mathrm{~K}$ produces too narrow spectra compared to the experiments, while the Q-sampled spectrum delivers satisfactory results. With an overheated T sampling, the spectrum is better, although still inferior to that obtained with $\mathrm{Q}$ sampling.

- Qualitatively, T and Q samplings lead to similar results in the dynamics. After excitation into $\mathrm{S}_{3}-\mathrm{S}_{6}$ manifold $\left(\pi \pi^{*}\right)$, the $\mathrm{S}_{2}$ and $\mathrm{S}_{1}$ states are populated under $30 \mathrm{fs}$, and the ground state is reached between 90 and a 148 fs through two types of $\mathrm{S}_{1} / \mathrm{S}_{0}$ intersections caused either by $\mathrm{H}$ elimination or by ring distortion.

- Quantitatively, dynamics stating from Q sampling is faster than starting from T by few tens of femtoseconds. When compared to the experimental results, Q sampling performs better in terms of time constants and distribution of reaction pathways. For instance, the time for $\mathrm{H}$ fragments formation is $64 \mathrm{fs}$ with $\mathrm{Q}$ and $100 \mathrm{fs}$ with $\mathrm{T}$, while the experimental value ${ }^{17}$ is $52 \pm 12$ fs.

Based on these points, quantum samplings based on Wigner distributions are clearly superior to thermal samplings, and they should be preferred for creating initial conditions for spectrum and excited-state dynamics simulations. Cases where Q sampling is computationally too demanding may be treated with hybrid methods.

Although we do not discuss this aspect here, we would like to remark that the exceptionally narrow ensemble distribution arising from $\mathrm{MM}$ and $\mathrm{BO}$ trajectories may have impact on ground-state investigations as well. This may be especially relevant when the ensemble is used to evaluate the probabilities of reaching transition states. Clearly, geometries and velocities in thermal ensembles 
populate a too restricted region of the phase space, which may lead to an overestimation of the time to reach transition states nearby.

The present simulations have been performed using $\mathrm{ADC}(2)$ method with the nuclear ensemble approach for spectrum simulations and surface hopping for dynamics. Compared to the experiments, $\mathrm{ADC}(2)$ provided excellent results for vertical excitations and very satisfactory results for spectrum simulations. It has also been able to describe the reaction path split of population during the dynamics between $\mathrm{H}$ elimination and ring distortion, a feature expected from photodissociation experiments, which previous simulations based on TDDFT and MRCI missed.

Finally, the present dynamics simulations for pyrrole reinforces the importance of further experiments on this molecule to take into account the substantial amount of internal conversion to the ground state occurring at ring-distorted pathways rather than at H-elimination pathways. The strong variations on the ionization potential along the main reaction coordinates in the excited states should also be considered.

\section{Acknowledgements}

This work has been carried out thanks to the support of the A*MIDEX grant ( $\mathrm{n}^{\circ}$ ANR-11-IDEX0001-02) funded by the French Government «Investissements d'Avenir » program. The authors thank Dr. Oliver Weingart, Dr. Michael Stenrup, Dr. Volker Haigis, and Prof. Rodolphe Vuilleumier for discussions.

\section{References}

1. Wu, G.; Neville, S. P.; Schalk, O.; Sekikawa, T.; Ashfold, M. N. R.; Worth, G. A.; Stolow, A. The Journal of Chemical Physics 2015, 142, 074302.

2. Jagau, T.-C.; Gauss, J. Chemical Physics 2012, 401, 73-87.

3. Li, X.; Paldus, J. The Journal of Physical Chemistry A 2010, 114, 8591-8600.

4. Shen, J.; Li, S. The Journal of Chemical Physics 2009, 131, 174101.

5. Evangelista, F. A.; Simmonett, A. C.; Allen, W. D.; Schaefer, H. F.; Gauss, J. The Journal of Chemical Physics 2008, 128, 124104.

6. King, R. A. The Journal of Physical Chemistry A 2008, 112, 5727-5733.

7. Burcl, R.; Carter, S.; Handy, N. C. Physical Chemistry Chemical Physics 2004, 6, 340-343.

8. Köppel, H.; Gromov, E. V.; Trofimov, A. B. Chemical Physics 2004, 304, 35-49. 
9. Wan, J.; Meller, J.; Hada, M.; Ehara, M.; Nakatsuji, H. Journal of Chemical Physics 2000, $113,7853-7866$.

10. Christiansen, O.; Gauss, J.; Stanton, J. F.; Jorgensen, P. Journal of Chemical Physics 1999, $111,525-537$.

11. Frank, I.; Damianos, K. Journal of Chemical Physics 2007, 126, 125105.

12. Pastore, M.; Angeli, C.; Cimiraglia, R. Chemical Physics Letters 2006, 422, 522-528.

13. Celani, P.; Werner, H. J. Journal of Chemical Physics 2003, 119, 5044-5057.

14. Sobolewski, A. L.; Domcke, W.; Dedonder-Lardeux, C.; Jouvet, C. Physical Chemistry Chemical Physics 2002, 4, 1093-1100.

15. Blank, D. A.; North, S. W.; Lee, Y. T. Chemical Physics 1994, 187, 35-47.

16. Montero, R.; Ovejas, V.; Fernández-Fernández, M.; Peralta Conde, Á.; Longarte, A. The Journal of Chemical Physics 2014, 141, 014303.

17. Roberts, G. M.; Williams, C. A.; Yu, H.; Chatterley, A. S.; Young, J. D.; Ullrich, S.; Stavros, V. G. Faraday Discussions 2013, 163, 95-116.

18. Liu, B.; Wang, Y.; Wang, L. The Journal of Physical Chemistry A 2012, 116, 111-118.

19. Montero, R.; Peralta Conde, Á.; Ovejas, V.; Fernández-Fernández, M.; Castaño, F.; Vázquez de Aldana, J. R.; Longarte, A. The Journal of Chemical Physics 2012, 137, 064317.

20. Wei, J.; Riedel, J.; Kuczmann, A.; Renth, F.; Temps, F. Faraday Discussions 2004, 127, $267-$ 282.

21. Lippert, H.; Ritze, H. H.; Hertel, I. V.; Radloff, W. Chemphyschem 2004, 5, 1423-1427.

22. Wei, J.; Kuczmann, A.; Riedel, J.; Renth, F.; Temps, F. Physical Chemistry Chemical Physics 2003, 5, 315-320.

23. van den Brom, A. J.; Kapelios, M.; Kitsopoulos, T. N.; Nahler, N. H.; Cronin, B.; Ashfold, M. N. R. Physical Chemistry Chemical Physics 2005, 7, 892-899.

24. Cronin, B.; Nix, M. G. D.; Qadiri, R. H.; Ashfold, M. N. R. Physical Chemistry Chemical Physics 2004, 6, 5031-5041.

25. Palmer, M. H.; Walker, I. C.; Guest, M. F. Chemical Physics 1998, 238, 179-199.

26. Palmer, M. H.; Wilson, P. J. Molecular Physics 2003, 101, 2391-2408.

27. Roos, B. O.; Malmqvist, P. A.; Molina, V.; Serrano-Andrés, L.; Merchán, M. Journal of Chemical Physics 2002, 116, 7526-7536.

28. Trofimov, A. B.; Köppel, H.; Schirmer, J. Journal of Chemical Physics 1998, 109, 1025-1040.

29. Faraji, S.; Vazdar, M.; Reddy, V. S.; Eckert-Maksic, M.; Lischka, H.; Köppel, H. The Journal of Chemical Physics 2011, 135, 154310.

30. Lan, Z.; Domcke, W. Chemical Physics 2008, 350, 125-138.

31. Lan, Z.; Dupays, A.; Vallet, V.; Mahapatra, S.; Domcke, W. Journal of Photochemistry and Photobiology a-Chemistry 2007, 190, 177-189. 
32. Vallet, V.; Lan, Z. G.; Mahapatra, S.; Sobolewski, A. L.; Domcke, W. Journal of Chemical Physics 2005, 123, 144307.

33. Neville, S. P.; Worth, G. A. The Journal of Chemical Physics 2014, 140, 034317.

34. Saita, K.; Nix, M. G. D.; Shalashilin, D. V. Physical Chemistry Chemical Physics 2013, 15, 16227-16235.

35. Sapunar, M.; Ponzi, A.; Chaiwongwattana, S.; Malis, M.; Prlj, A.; Decleva, P.; Doslic, N. Physical Chemistry Chemical Physics 2015, 17, 19012-19020.

36. Vazdar, M.; Eckert-Maksic, M.; Barbatti, M.; Lischka, H. Molecular Physics 2009, 107, 845 854.

37. Barbatti, M.; Vazdar, M.; Aquino, A. J. A.; Eckert-Maksic, M.; Lischka, H. Journal of Chemical Physics 2006, 125, 164323.

38. Barbatti, M.; Pittner, J.; Pederzoli, M.; Werner, U.; Mitrić, R.; Bonačić-Koutecký, V.; Lischka, H. Chemical Physics 2010, 375, 26-34.

39. Sellner, B.; Barbatti, M.; Lischka, H. Journal of Chemical Physics 2009, 131, 024312.

40. Klaffki, N.; Weingart, O.; Garavelli, M.; Spohr, E. Physical Chemistry Chemical Physics 2012, 14, 14299-14305.

41. Wigner, E. Physical Review 1932, 40, 749-759.

42. Lukes, V.; Solc, R.; Barbatti, M.; Lischka, H.; Kauffmann, H. F. Journal of Theoretical \& Computational Chemistry 2010, 9, 249-263.

43. Ruckenbauer, M.; Barbatti, M.; Müller, T.; Lischka, H. The Journal of Physical Chemistry A 2013, 117, 2790-2799.

44. Ruckenbauer, M.; Barbatti, M.; Muller, T.; Lischka, H. Journal of Physical Chemistry A 2010, 114, 6757-6765.

45. Trofimov, A. B.; Schirmer, J. Journal of Physics B: Atomic, Molecular and Optical Physics 1995, 28, 2299-2324.

46. Schirmer, J. Physical Review A 1982, 26, 2395-2416.

47. Hättig, C.; Köhn, A. Journal of Chemical Physics 2002, 117, 6939-6951.

48. Hättig, C.; Weigend, F. Journal of Chemical Physics 2000, 113, 5154-5161.

49. Dunning Jr., T. H. The Journal of Chemical Physics 1989, 90, 1007-1023.

50. Crespo-Otero, R.; Barbatti, M. Theoretical Chemistry Accounts 2012, 131, 1237.

51. Barbatti, M. Willey Interdisciplinary Reviews: Computational Molecular Sciences 2011, 1, 620-633.

52. Tully, J. C. Journal of Chemical Physics 1990, 93, 1061-1071.

53. Granucci, G.; Persico, M. Journal of Chemical Physics 2007, 126, 134114-134111.

54. Plasser, F.; Crespo-Otero, R.; Pederzoli, M.; Pittner, J.; Lischka, H.; Barbatti, M. Journal of Chemical Theory and Computation 2014, 10, 1395-1405.

55. Hammes-Schiffer, S.; Tully, J. C. Journal of Chemical Physics 1994, 101, 4657-4667. 
56. Ahlrichs, R.; Bär, M.; Häser, M.; Horn, H.; Kölmel, C. Chem. Phys. Lett. 1989, 162, 165-169.

57. Barbatti, M.; Ruckenbauer, M.; Plasser, F.; Pittner, J.; Granucci, G.; Persico, M.; Lischka, H. Willey Interdisciplinary Reviews: Computational Molecular Sciences 2014, 4, 26-33.

58. Barbatti, M.; Granucci, G.; Ruckenbauer, M.; Plasser, F.; Crespo-Otero, R.; Pittner, J.; Persico, M.; Lischka, H. NEWTON-X: a package for Newtonian dynamics close to the crossing seam 2013, www.newtonx.org.

59. Levine, B. G.; Coe, J. D.; Martínez, T. J. The Journal of Physical Chemistry B 2008, 112, 405-413.

60. Spek, A. L. Journal of Applied Crystallography 2003, 36, 7-13.

61. Andersen, H. C. Journal of Chemical Physics 1980, 72, 2384-2393.

62. Case, D. A.; Darden, T. A.; T.E. Cheatham, I.; Simmerling, C. L.; Wang, J.; Duke, R. E.; Luo, R.; Walker, R. C.; Zhang, W.; Merz, K. M.; Roberts, B.; Wang, B.; Hayik, S.; Roitberg, A.; Seabra, G.; Kolossváry, I.; Wong, K. F.; Paesani, F.; Vanicek, J.; Liu, J.; Wu, X.; Brozell, S. R.; Steinbrecher, T.; Gohlke, H.; Cai, Q.; Ye, X.; Wang, J.; Hsieh, M.-J.; Cui, G.; Roe, D. R.; Mathews, D. H.; Seetin, M. G.; Sagui, C.; Babin, V.; Luchko, T.; Gusarov, S.; Kovalenko, A.; Kollman, P. A. Amber 11, University of California, San Francisco 2010.

63. Yu, W.; He, X.; Vanommeslaeghe, K.; MacKerell, A. D. Journal of Computational Chemistry 2012, 33, 2451-2468.

64. Vanommeslaeghe, K.; Hatcher, E.; Acharya, C.; Kundu, S.; Zhong, S.; Shim, J.; Darian, E.; Guvench, O.; Lopes, P.; Vorobyov, I.; Mackerell, A. D. Journal of Computational Chemistry 2010, 31, 671-690.

65. Bergsma, J. P.; Berens, P. H.; Wilson, K. R.; Fredkin, D. R.; Heller, E. J. Journal of Physical Chemistry 1984, 88, 612-619.

66. Petit, A. S.; Subotnik, J. E. Journal of Chemical Theory and Computation 2015, 11, 43284341.

67. Barbatti, M.; Lischka, H.; Salzmann, S.; Marian, C. M. Journal of Chemical Physics 2009, $130,034305$.

68. Cremer, D.; Pople, J. A. Journal of the American Chemical Society 1975, 97, 1354-1358.

69. Desouter-Lecomte, M.; Lorquet, J. C. The Journal of Chemical Physics 1979, 71, 4391-4403.

70. Barbatti, M. Physical Chemistry Chemical Physics 2011, 13, 4686-4692.

71. Xie, Z.; Bowman, J. M. The Journal of Physical Chemistry A 2006, 110, 5446-5449. 